\title{
gु
}

\section{Dynamical Quasicondensation of Hard-Core Bosons at Finite Momenta}

\author{
L. Vidmar, ${ }^{1,2}$ J. P. Ronzheimer, ${ }^{2,3}$ M. Schreiber, ${ }^{2,3}$ S. Braun, ${ }^{2,3}$ S. S. Hodgman, ${ }^{2,3}$ S. Langer, ${ }^{4}$ \\ F. Heidrich-Meisner, ${ }^{1,2}$ I. Bloch, ${ }^{2,3}$ and U. Schneider, ${ }^{2,3,5}$ \\ ${ }^{1}$ Arnold Sommerfeld Center for Theoretical Physics, \\ Ludwig-Maximilians-Universität München, D-80333 München, Germany \\ ${ }^{2}$ Department of Physics, Ludwig-Maximilians-Universität München, D-80333 München, Germany \\ ${ }^{3}$ Max-Planck-Institut für Quantenoptik, D-85748 Garching, Germany \\ ${ }^{4}$ Department of Physics and Astronomy, University of Pittsburgh, Pittsburgh, Pennsylvania 15213, USA \\ ${ }^{5}$ Cavendish Laboratory, University of Cambridge, J. J. Thomson Avenue, Cambridge CB3 OHE, United Kingdom
}

(Received 22 May 2015; published 19 October 2015)

\begin{abstract}
Long-range order in quantum many-body systems is usually associated with equilibrium situations. Here, we experimentally investigate the quasicondensation of strongly interacting bosons at finite momenta in a far-from-equilibrium case. We prepare an inhomogeneous initial state consisting of one-dimensional Mott insulators in the center of otherwise empty one-dimensional chains in an optical lattice with a lattice constant $d$. After suddenly quenching the trapping potential to zero, we observe the onset of coherence in spontaneously forming quasicondensates in the lattice. Remarkably, the emerging phase order differs from the ground-state order and is characterized by peaks at finite momenta $\pm(\pi / 2)(\hbar / d)$ in the momentum distribution function.
\end{abstract}

DOI: 10.1103/PhysRevLett.115.175301

PACS numbers: 67.85.-d, 05.30.Jp, 05.60.Gg, 05.70.Ln

The nonequilibrium dynamics of quantum manybody systems constitutes one of the most challenging and intriguing topics in modern physics. Generically, interacting many-body systems are expected to relax towards equilibrium and eventually thermalize [1,2]. This standard picture, however, does not always apply.

In open or driven systems, one fascinating counterexample is the emergence of novel steady states with far-fromequilibrium long-range order, i.e., order that is absent in the equilibrium phase diagram. This includes lasers [3], where strong incoherent pumping gives rise to a coherent emission and driven ultracold atom systems [4]. The emergence of order far from equilibrium is also studied in condensed matter systems [5] and optomechanical systems [6].

In recent years, closed quantum systems without any coupling to an environment have come into the focus of experimental and theoretical research. Experimental examples range from ultracold atoms [7-15] to quark-gluon plasmas in heavy ion collisions [16]. In closed nondriven systems, two famous examples of the absence of thermalization [8,9,14] are many-body-localized [17-19] and integrable systems [20]. These peculiar systems allow for nonergodic dynamics and novel quantum phenomena.

Spontaneously emerging order is, in general, associated with equilibrium states at low temperatures. The canonical example is the emergence of (quasi-) long-range phase coherence when cooling an ideal Bose gas into a BoseEinstein (quasi-) condensate [21,22]. In this case, thermodynamics ensures that, for positive temperatures [23], the single-particle ground state becomes macroscopically occupied and thereby dictates the emerging order. Even in studies of the nonequilibrium dynamics at quantum phase transitions [24], the emergence of coherence is typically associated with gently crossing the transition from an unordered into an ordered state, and the strongest correlations and largest coherence lengths appear in the adiabatic limit [25,26].

Here, in contrast, we study a condensation phenomenon of strongly interacting lattice bosons far from equilibrium. After a sudden quantum quench, we experimentally observe the spontaneous emergence of a long-lived phase order that is markedly different from the equilibrium order (cf. Fig. 1). To this end, we prepare a density-one Mott

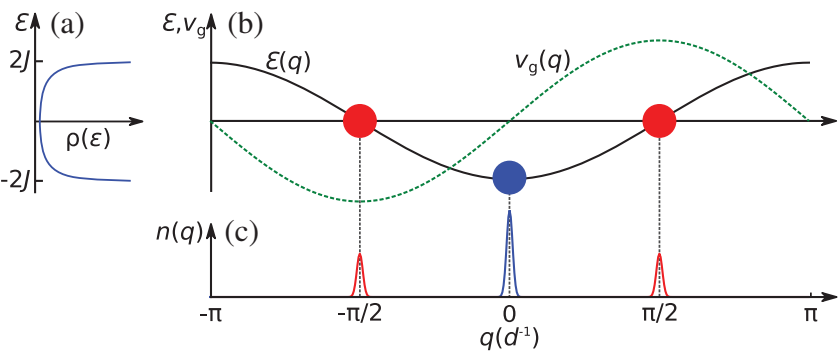

FIG. 1 (color). Quasicondensation of bosons. (a) Density of states $\rho(\epsilon)$ of a homogeneous 1D lattice. (b) Dispersion $\varepsilon(q)$ (solid line) and group velocity $v_{g}(q)$ (dotted line) versus quasimomentum $q$. (c) Sketch of quasimomentum distribution $n(q)$ : In equilibrium, $1 \mathrm{D}$ bosons quasicondense at the minimum of the band at $q=0$, while in a sudden expansion the quasicondensation of hard-core bosons occurs at $\hbar q= \pm(\pi / 2)(\hbar / d)$. This quasimomentum lies in the middle of the spectrum and is consistent with the vanishing energy per particle of this closed many-body system. 
insulator of strongly interacting bosons in the center of a three-dimensional (3D) optical lattice. Next, we transform the system into an array of independent one-dimensional (1D) chains entering the regime of integrable hard-core bosons (HCBs). By suddenly quenching the confining potential along the chains to zero, we induce a sudden expansion of the cloud in a homogeneous lattice $[12,13,15,27]$ with a lattice constant $d$ and detect the formation of a non-ground-state phase profile as a dynamical emergence of peaks at momenta $\hbar k= \pm(\pi / 2)(\hbar / d)$, halfway between the middle and the edge of the Brillouin zone in time-of-flight (TOF) distributions. This finitemomentum quasicondensation was first discussed by Rigol and Muramatsu [28] (see, also, Refs. [29-33]) but has not been studied experimentally so far.

Ideal case.-The idealized setup to study finitemomentum quasicondensates is shown in Fig. 2. We consider the Hamiltonian $H=-J \sum_{j}\left(\hat{a}_{j+1}^{\dagger} \hat{a}_{j}+\right.$ H.c. $)$, where $\hat{a}_{j}^{\dagger}$ creates a HCB on site $j$ of a $1 \mathrm{D}$ lattice. The infinitely large on-site repulsion is accounted for by the hard-core constraint $\left(\hat{a}_{j}^{\dagger}\right)^{2}=0$. The initial state is a product state $\left|\psi_{0}\right\rangle=\prod_{j \in L_{0}} \hat{a}_{j}^{\dagger}|\emptyset\rangle$, completely filling the central $L_{0}$ sites of an otherwise empty and infinitely large 1D lattice. This initial state consists of $N=L_{0}$ localized particles with a flat quasimomentum distribution and contains no
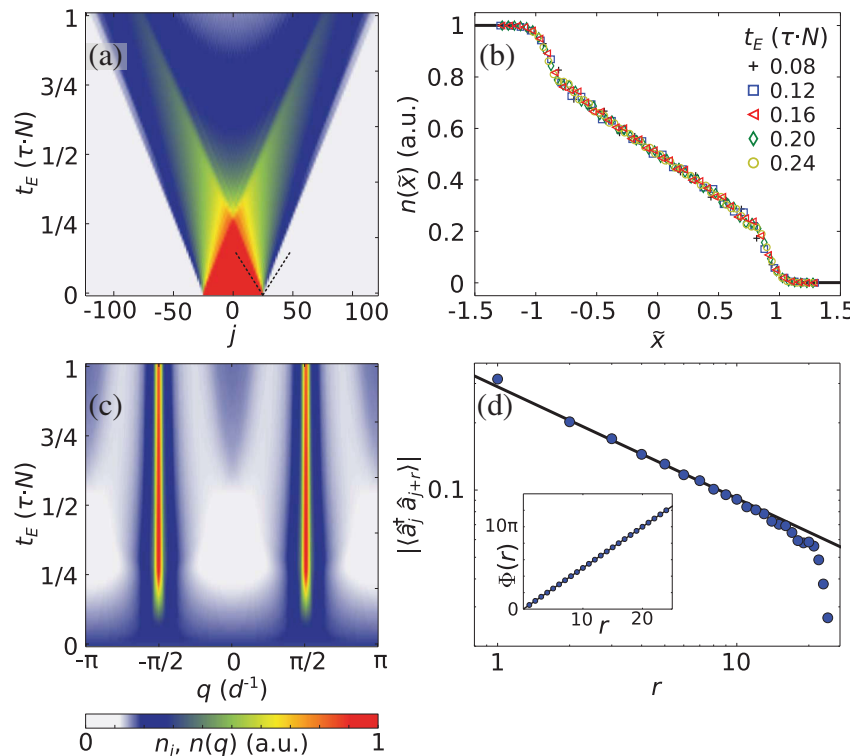

FIG. 2 (color). Finite-momentum quasicondensation under ideal conditions [28]. We consider $N=50$ initially localized HCBs. (a) Density $n_{j}$ as a function of time. (b) Density as a function of the rescaled coordinate $\tilde{x}=(j-25.5) /\left(2 t_{E} / \tau\right)$ in the region bounded by the dashed lines in (a). For $\tilde{x} \leq 1$, the data collapse to the scaling solution [34] $n(\tilde{x})=\arccos (\tilde{x}) / \pi$. (c) Quasimomentum distribution $n(q)$ as a function of time. (d) One-particle correlations at $t_{E}=0.24 N \tau$. Main panel: $\left|\left\langle\hat{a}_{j}^{\dagger} \hat{a}_{j+r}\right\rangle\right|$ at $j=26$ (circles) and $\mathcal{A}(r)=\alpha / \sqrt{r}$ (line) with $\alpha=0.29$ [35]. Inset: Phase pattern $\Phi(r)$. off-diagonal correlations, i.e., $\left\langle\hat{a}_{j}^{\dagger} \hat{a}_{j+r}\right\rangle=0$ for $r \neq 0$. Surprisingly, the quasimomentum distribution $n(q)=$ $\frac{1}{L} \sum_{j, l} e^{-i q(j-l) d}\left\langle\hat{a}_{j}^{\dagger} \hat{a}_{l}\right\rangle$ develops singularities at finite quasimomenta $\hbar q= \pm(\pi / 2)(\hbar / d)$ during the expansion $\left(t_{E}>0\right)$. As shown in Fig. 2(c), these singularities correspond to the emergence of power-law correlations

$$
\left\langle\hat{a}_{j}^{\dagger} \hat{a}_{j+r}\right\rangle=\mathcal{A}(r) e^{i \Phi(r)}, \quad \mathcal{A}(r) \sim r^{-(1 / 2)}, \quad \Phi(r)= \pm \frac{\pi}{2} r
$$

in each half of the expanding cloud shown in Fig. 2(d). These power-law correlations justify the name quasicondensate [28]. Curiously, the exponent $1 / 2$ equals the ground-state exponent [28,30], even though the system is far away from equilibrium, with the energy per particle being much higher than in the ground state. In contrast to the ground state, the correlations show a running phase pattern $\Phi(r)$ with a phase difference of $\pm \pi / 2$ between neighboring lattice sites, giving rise to peaks at finite quasimomenta. Coherence and quasicondensation emerge independently in the left- and right-moving halves of the cloud, corresponding to two macroscopically occupied degenerate eigenstates of the one-particle density matrix $\left\langle\hat{a}_{j}^{\dagger} \hat{a}_{l}\right\rangle$ that have spatial support in the left- or right-moving cloud, respectively [28]. This quasicondensation at finite quasimomenta can equivalently be seen as quasicondensation at $q=0$ in the respective comoving frames [32].

In one dimension, HCBs can be exactly mapped to noninteracting spinless fermions via the Jordan-Wigner transformation [36]. By virtue of this mapping, the density $n_{j}=\left\langle\hat{a}_{j}^{\dagger} \hat{a}_{j}\right\rangle$ of HCBs is identical to that of free fermions for all times, whereas the same is not true for the quasimomentum distribution [37,38]. While the occupations of fermionic quasimomenta are constants of motion, the nonlocal phase factors in the Jordan-Wigner transformation give rise to the intricate momentum dynamics studied here. In the ideal case, the dynamical quasicondensates form over a time scale $t_{E}^{*} \sim 0.3 N \tau[28,33]$, where $N$ is the number of particles in the initial state, and $\tau=\hbar / J$ denotes the tunneling time. For very long times, $n(q)$ slowly decays back to its original flat form as a consequence of the dynamical fermionization mechanism $[33,39,40]$.

The dynamical quasicondensation at finite momenta is an example of a more general emergence of coherence in a sudden expansion. For instance, interacting fermions described by the Fermi-Hubbard model exhibit groundstate correlations in the transient dynamics as well [32]. Furthermore, there is a close connection to quantum magnetism, as can be seen by mapping $\mathrm{HCBs}$ to a spin$1 / 2 X X$ chain: the transient dynamics in each half of the expanding cloud of $\mathrm{HCBs}$ is equivalent to the melting of a domain-wall state [34,41-49] of the form $\left|\psi_{0}\right\rangle=$ $|\uparrow \ldots \uparrow \uparrow \downarrow \downarrow \ldots \downarrow\rangle$. For this problem, a scaling solution exists 
[34], which also applies to the sudden expansion at times $t<t_{E}^{*}$. As a consequence, the densities $n_{j}$ measured at different times collapse onto a single curve, as shown in Fig. 2(b). Furthermore, for spin-1/2 $X X$ chains, the emergence of power-law decaying transverse spin correlations modulated with a phase of $\pi / 2$ has been derived analytically [44]. An interesting perspective into the emergence of coherence results from noticing that both our expanding bosons and the melting domain walls realize current-carrying nonequilibrium steady states (see Ref. [50] for a discussion).

Experimental setup and results.-The experimental setup is identical to that employed in our previous experiment on in situ density dynamics [13]. We load a BoseEinstein condensate of approximately $10^{5}{ }^{39} \mathrm{~K}$ atoms from a crossed optical dipole trap into a blue-detuned 3D optical lattice with a lattice depth of $V_{0} \approx 20 E_{r}$, where $E_{r}=$ $h^{2} /\left(2 m \lambda^{2}\right)$ denotes the recoil energy with atomic mass $m$ and lattice laser wavelength $\lambda \approx 737 \mathrm{~nm}$. During the loading of the lattice, we use a magnetic Feshbach resonance to induce strong repulsive interactions between the atoms, suppressing the formation of doubly occupied sites [13]. This results in a large density-one Mott insulator in the center of the cloud. We hold the atoms in the deep lattice for a $20 \mathrm{~ms}$ dephasing period, during which residual correlations between lattice sites are mostly lost such that the atoms essentially become localized to individual lattice sites [67]. The expansion is initiated at $t_{E}=0$ by simultaneously lowering the lattice depth along the expansion axis in $150 \mu$ s to $V_{0}^{x} \approx 8 E_{r}$ (setting $J \approx h \times 300 \mathrm{~Hz}, \tau \approx 0.5 \mathrm{~ms}$ ) and reducing the strength of the optical dipole trap to exactly compensate the anticonfinement of the bluedetuned lattice beams. This creates a flat potential along the expansion direction. Figures 3(b)-3(e) show the ballistic expansion of the in situ density [13] monitored using absorption imaging. During the deep lattice period, the magnetic field is changed to tune the on-site interaction strength during the expansion to $U=20 \mathrm{~J}$. We have numerically verified that the essential features of dynamical quasicondensation are still present for this value of $U / J$, with a shift of the peak position by $\approx 10 \%$ towards smaller values $[31,50]$.

In order to measure the momentum distribution as a function of expansion time $t_{E}$, we employ an adapted TOF imaging technique. Directly before shutting off all lattice and trapping potentials, we rapidly increase the lattice intensity along the expansion axis for $5 \mu$ s to a depth of $33 E_{r}$. This time is too short to affect correlations between different sites and the momentum distribution. Nonetheless, it results in a narrowing of the Wannier functions, which leads to a broadening of the Wannier envelope in the TOF density distribution and thereby facilitates the observation of higher-order peaks [50].

Figures 3(f)-3(i) contain the main result of our experiment, namely, the TOF density distributions, which correspond approximately to the momentum distribution, taken at different expansion times $t_{E}$. In Fig. 3(f), the TOF sequence was initiated at $t_{E}=0$, i.e., directly after initiating the expansion. We observe a central peak at $k=0$ and two higher-order peaks at $\hbar k= \pm(2 \pi)(\hbar / d)$, indicating a weak residual $k=0$ coherence probably resulting from an imperfect state preparation. During the expansion,
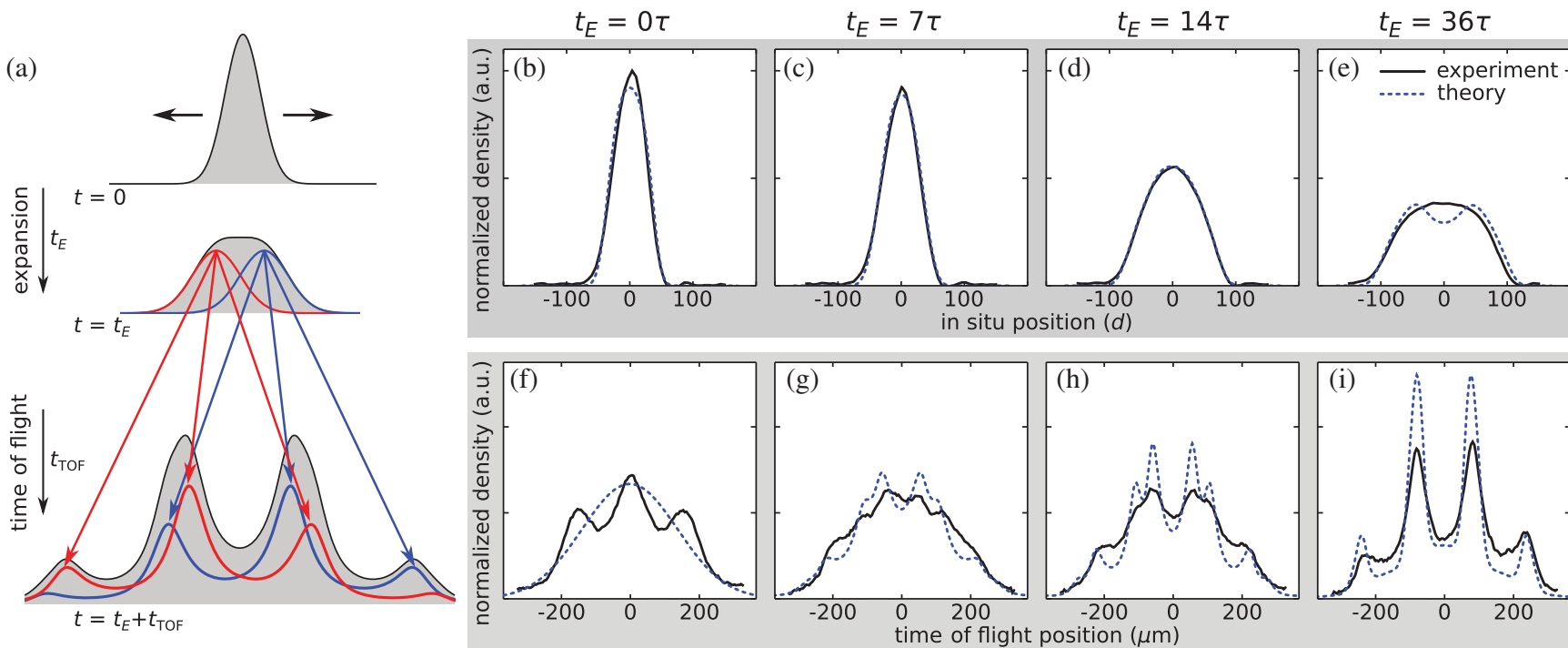

FIG. 3 (color). Dynamics during sudden expansion and time-of-flight sequence. (a) Sketch of the experimental sequence: We start with a trapped gas that is released from the initial confinement at $t=0$ (top) and then expands for a time $t_{E}$ in the optical lattice (middle). At $t_{E}$, all potentials are removed and the distribution is measured after a finite time-of-flight time $t_{\mathrm{TOF}}$ (bottom). (b)-(e): In situ density distributions during the sudden expansion integrated along the $y$ and $z$ axes. The lattice constant corresponds to $d=\lambda / 2 \approx 368 \mathrm{~nm}$. (f)-(i): TOF density distributions taken at $t_{\mathrm{TOF}}=6 \mathrm{~ms}$ for the same $t_{E}$ as in (b)-(e). All measured density distributions are averaged over 9 to 11 experimental realizations. 
however, the momentum distribution changes fundamentally and the remnants of the initial coherence quickly vanish. Instead, new peaks at finite momenta are formed. These peaks directly signal the spontaneous formation of a different phase order. This is best seen in Fig. 3(i) at $t_{E}=36 \tau$, where the finite-momenta peaks are clearly established. The observed peak positions correspond to the expected momenta close to $\hbar k= \pm(\pi / 2)(\hbar / d)$ [50]. In addition, Figs. $3(\mathrm{~g})$ and $3(\mathrm{~h})$ taken at $t_{E}=7 \tau$ and $14 \tau$, respectively, hint at a fine structure of the emerging peaks. This structure is a consequence of the finite TOF time $t_{\mathrm{TOF}}=6 \mathrm{~ms}$, which results in the TOF distributions being a convolution of real-space and momentum-space densities. We sketch this situation in Fig. 3(a). As discussed before, the peaks in $n(k)$ close to $\hbar k=-(\pi / 2)(\hbar / d)$ and $+(\pi / 2)(\hbar / d)$ originate from the left- and right-moving portions of the cloud, respectively. Because of the finite $t_{\mathrm{TOF}}$, the higher-order peak of the left-moving cloud with momentum $(-\pi / 2+2 \pi)(\hbar / d)$ and the main peak of the right-moving cloud with momentum $(\pi / 2)(\hbar / d)$ (and vice versa) may overlap in the TOF data. A perfect overlap gives rise to single sharp peaks such as the ones present in the data shown in Fig. 3(i), while shorter expansion times, as shown in Figs. 3(g) and 3(h), result in a partial overlap and additional structure (see Ref. [50] for details).

Comparison with exact time evolution.-We numerically model the dynamics of 1D HCBs for realistic conditions: (i) The experimental setup consists of many isolated 1D chains, which are not equivalent due to the 3D harmonic confinement. Experimentally, we can only measure an ensemble average over all tubes. (ii) Both the finite temperature of the original 3D Bose-Einstein condensate as well as nonadiabaticities during the lattice loading result in a finite entropy, and thereby holes, in the initial state. We, therefore, average the results over different initial product states drawn from a thermal ensemble of a harmonically trapped 3D gas of HCBs in the atomic limit [50]. Chemical potential and temperature were calibrated to reproduce the experimental atom number and an average entropy per particle of $1.2 k_{B}$ [68], thereby leaving no free parameters for the simulations. To test the consistency of the approach, we compare the average density $n_{j}$ during the expansion with the in situ images in Figs. 3(b)-3(e) and find a good agreement. In addition, the time evolution of the half width at half maximum of the density distribution shown in Fig. S4 of Ref. [50] is consistent with the ballistic dynamics as previously measured in the same experimental setup [13].

Since the momentum distribution is experimentally measured at a finite $t_{\mathrm{TOF}}$, we explicitly calculate the TOF density distributions without employing the far-field approximation [50,69] and compare the results to the experimental data in Figs. 3(f)-3(i). Remarkably, the positions and the structure of the peaks agree very well between experiment and theory, thereby supporting our two main results: the central peaks indeed correspond to a large occupation of quasimomenta close to $\hbar q= \pm(\pi / 2)(\hbar / d)$, i.e., to a bunching of particles around the fastest group velocities in the middle of the single-particle spectrum. In addition, the fine structure visible for intermediate expansion times [cf. Figs. 3(g) and 3(h)], which becomes more apparent in the experiment when comparing different $t_{\mathrm{TOF}}$ (cf. Fig. S2 in Ref. [50]), directly confirms the independent emergence of coherence in the left- and right-moving portions of the cloud. Compared to the ideal case, the presence of holes in the initial state causes a reduced visibility of the TOF density distributions [50]. Moreover, the finite initial entropy gives rise to a crossover of oneparticle correlations from a power-law decay at short distances to a more rapid decay at long distances [50], similar to the effect of a finite temperature [70] in equilibrium.

We attribute the discrepancies between experimental and numerical results at short times [see Fig. 3(f)] to the weak residual $k=0$ coherence in the initial state. Additional discrepancies may arise because of a small admixture $(\lesssim 5 \%)$ of doublons in the initial state $[13,15]$ as well as small residual potentials, yet we conclude that these play a minor role [50]. Compared to the previously studied time dependence of density distributions and expansion velocities [13], the momentum distribution is more sensitive to such imperfections [50]. Performing a similar experiment with a single 1D system would allow the predicted scaling of $t_{E}^{*}$ and the maximum peak height with atom number [28] to be experimentally tested.

Conclusions and outlook.-We have reported experimental evidence for a far-from-equilibrium quasicondensation at finite momenta of expanding $1 \mathrm{D} \mathrm{HCBs}$ in an optical lattice. The expanding particles bunch at quasimomenta close to $\pm(\pi / 2)(\hbar / d)$ and the analysis of TOF distributions demonstrates the existence of two independent sources of coherence.

Whether such dynamical condensation persists in higher-dimensional systems constitutes an open problem, given that the existing theoretical results are based on exact diagonalization of small systems [71] or timedependent Gutzwiller simulations [72]. Both future experiments or advanced numerical methods (see, e.g., Refs. [73,74]) could help clarify this question. More generally, our results raise the question of whether this type of spontaneously emerging coherence is limited to integrable systems and whether genuinely far-fromequilibrium order can also occur in generic closed many-body systems.

We thank F. Essler, A. Mitra, and M. Rigol for helpful discussions. We acknowledge support from the Deutsche Forschungsgemeinschaft (DFG) through FOR 801, and the EU (AQuS, UQUAM). This work was supported in part by National Science Foundation Grant No. PHYS-1066293 and the hospitality of the Aspen Center for Physics. L. V. was supported by the Alexander von Humboldt Foundation. 
[1] M. Rigol, V. Dunjko, and M. Olshanii, Thermalization and its mechanism for generic isolated quantum systems, Nature (London) 452, 854 (2008).

[2] A. Polkovnikov, K. Sengupta, A. Silva, and M. Vengalattore, Nonequilibrium dynamics of closed interacting quantum systems, Rev. Mod. Phys. 83, 863 (2011).

[3] A. Chiocchetta, A. Gambassi, and I. Carusotto, Laser operation and Bose-Einstein condensation: Analogies and differences, arXiv:1503.02816.

[4] D. Vorberg, W. Wustmann, R. Ketzmerick, and A. Eckardt, Generalized Bose-Einstein Condensation into Multiple States in Driven-Dissipative Systems, Phys. Rev. Lett. 111, 240405 (2013).

[5] L. Stojchevska, I. Vaskivskyi, T. Mertelj, P. Kusar, D. Svetin, S. Brazovskii, and D. Mihailovic, Ultrafast switching to a stable hidden quantum state in an electronic crystal, Science 344, 177 (2014).

[6] M. Ludwig and F. Marquardt, Quantum Many-Body Dynamics in Optomechanical Arrays, Phys. Rev. Lett. 111, 073603 (2013).

[7] M. Greiner, O. Mandel, T. Hänsch, and I. Bloch, Collapse and revival of the matter wave field of a Bose-Einstein condensate, Nature (London) 419, 51 (2002).

[8] T. Kinoshita, T. Wenger, and D.S. Weiss, A quantum Newton's cradle, Nature (London) 440, 900 (2006).

[9] S. Hofferberth, I. Lesanovsky, B. Fisher, T. Schumm, and J. Schmiedmayer, Non-equilibrium coherence dynamics in one-dimensional Bose gases, Nature (London) 449, 324 (2007).

[10] S. Trotzky, Y.-A. Chen, A. Flesch, I. P. McCulloch, U. Schollwöck, J. Eisert, and I. Bloch, Probing the relaxation towards equilibrium in an isolated strongly correlated onedimensional Bose gas, Nat. Phys. 8, 325 (2012).

[11] M. Cheneau, P. Barmettler, D. Poletti, M. Endres, P. Schauß, T. Fukuhara, C. Gross, I. Bloch, C. Kollath, and S. Kuhr, Light-cone-like spreading of correlations in a quantum many-body system, Nature (London) 481, 484 (2012).

[12] U. Schneider, L. Hackermüller, J. P. Ronzheimer, S. Will, S. Braun, T. Best, I. Bloch, E. Demler, S. Mandt, D. Rasch, and A. Rosch, Breakdown of diffusion: From collisional hydrodynamics to a continuous quantum walk in a homogeneous Hubbard model, Nat. Phys. 8, 213 (2012).

[13] J. P. Ronzheimer, M. Schreiber, S. Braun, S. S. Hodgman, S. Langer, I. P. McCulloch, F. Heidrich-Meisner, I. Bloch, and U. Schneider, Expansion Dynamics of Interacting Bosons in Homogeneous Lattices in One and Two Dimensions, Phys. Rev. Lett. 110, 205301 (2013).

[14] T. Langen, R. Geiger, M. Kuhnert, B. Rauer, and J. Schmiedmayer, Local emergence of thermal correlations in an isolated quantum many-body system, Nat. Phys. 9, 640 (2013).

[15] L. Xia, L. A. Zundel, J. Carrasquilla, A. Reinhard, J. M. Wilson, M. Rigol, and D. S. Weiss, Quantum distillation and confinement of vacancies in a doublon sea, Nat. Phys. 11, 316 (2015).

[16] J. Berges, K. Boguslavski, S. Schlichting, and R. Venugopalan, Universality Far from Equilibrium: From Superfluid Bose Gases to Heavy-Ion Collisions, Phys. Rev. Lett. 114, 061601 (2015).
[17] R. Nandishore and D. A. Huse, Many body localization and thermalization in quantum statistical mechanics, Annu. Rev. Condens. Matter Phys. 6, 15 (2015).

[18] E. Altman and R. Vosk, Universal dynamics and renormalization in many-body-localized systems, Annu. Rev. Condens. Matter Phys. 6, 383 (2015).

[19] M. Schreiber, S. S. Hodgman, P. Bordia, H. P. Lüschen, M. H. Fischer, R. Vosk, E. Altman, U. Schneider, and I. Bloch, Observation of many-body localization of interacting fermions in a quasirandom optical lattice, Science 349, 842 (2015).

[20] M. Rigol, V. Dunjko, V. Yurovsky, and M. Olshanii, Relaxation in a Completely Integrable Many-Body Quantum System: An $A b$ Initio Study of the Dynamics of the Highly Excited States of 1D Lattice Hard-Core Bosons, Phys. Rev. Lett. 98, 050405 (2007).

[21] M. H. Anderson, J. R. Ensher, M. R. Matthews, C. E. Wieman, and E. A. Cornell, Observation of Bose-Einstein condensation in a dilute atomic vapor, Science 269, 198 (1995).

[22] K. B. Davis, M. O. Mewes, M. R. Andrews, N. J. van Druten, D. S. Durfee, D. M. Kurn, and W. Ketterle, BoseEinstein Condensation in a Gas of Sodium Atoms, Phys. Rev. Lett. 75, 3969 (1995).

[23] S. Braun, J. P. Ronzheimer, M. Schreiber, S. S. Hodgman, T. Rom, I. Bloch, and U. Schneider, Negative absolute temperature for motional degrees of freedom, Science 339, 52 (2013).

[24] S. Sachdev, Quantum Phase Transitions (Cambridge University Press, New York, 2011).

[25] D. Chen, M. White, C. Borries, and B. DeMarco, Quantum Quench of an Atomic Mott Insulator, Phys. Rev. Lett. 106, 235304 (2011).

[26] S. Braun, M. Friesdorf, S. S. Hodgman, M. Schreiber, J. P. Ronzheimer, A. Riera, M. del Rey, I. Bloch, J. Eisert, and U. Schneider, Emergence of coherence and the dynamics of quantum phase transitions, Proc. Natl. Acad. Sci. U.S.A. 112, 3641 (2015).

[27] A. Reinhard, J.-F. Riou, L. A. Zundel, D. S. Weiss, S. Li, A. M. Rey, and R. Hipolito, Self-Trapping in an Array of Coupled 1D Bose Gases, Phys. Rev. Lett. 110, 033001 (2013).

[28] M. Rigol and A. Muramatsu, Emergence of Quasicondensates of Hard-Core Bosons at Finite Momentum, Phys. Rev. Lett. 93, 230404 (2004).

[29] A. J. Daley, S. R. Clark, D. Jaksch, and P. Zoller, Numerical analysis of coherent many-body currents in a single atom transistor, Phys. Rev. A 72, 043618 (2005).

[30] M. Rigol and A. Muramatsu, Free expansion of impenetrable bosons on one-dimensional optical lattices, Mod. Phys. Lett. B 19, 861 (2005).

[31] K. Rodriguez, S. Manmana, M. Rigol, R. Noack, and A. Muramatsu, Coherent matter waves emerging from Mott insulators, New J. Phys. 8, 169 (2006).

[32] F. Heidrich-Meisner, M. Rigol, A. Muramatsu, A. E. Feiguin, and E. Dagotto, Ground-state reference systems for expanding correlated fermions in one dimension, Phys. Rev. A 78, 013620 (2008).

[33] L. Vidmar, S. Langer, I. P. McCulloch, U. Schneider, U. Schollwöck, and F. Heidrich-Meisner, Sudden expansion of Mott insulators in one dimension, Phys. Rev. B 88, 235117 (2013). 
[34] T. Antal, Z. Rácz, A. Rákos, and G. M. Schütz, Transport in the $X X$ chain at zero temperature: Emergence of flat magnetization profiles, Phys. Rev. E 59, 4912 (1999).

[35] A. A. Ovchinikov, Fisher-Hartwig conjecture and the correlators in $X Y$ spin chain, Phys. Lett. A 366, 357 (2007).

[36] M. A. Cazalilla, R. Citro, T. Giamarchi, E. Orignac, and M. Rigol, One dimensional bosons: From condensed matter systems to ultracold gases, Rev. Mod. Phys. 83, 1405 (2011).

[37] B. Paredes, A. Widera, V. Murg, O. Mandel, S. Fölling, I. Cirac, G. Shlyapnikov, T. H. Hänsch, and I. Bloch, Tonks-Girardeau gas of ultracold atoms in an optical lattice, Nature (London) 429, 277 (2004).

[38] T. Kinoshita, T. Wenger, and D. S. Weiss, Observation of a one-dimensional Tonks-Girardeau gas, Science 305, 1125 (2004).

[39] M. Rigol and A. Muramatsu, Fermionization in an Expanding 1D Gas of Hard-Core Bosons, Phys. Rev. Lett. 94, 240403 (2005).

[40] A. Minguzzi and D. M. Gangardt, Exact Coherent States of a Harmonically Confined Tonks-Girardeau Gas, Phys. Rev. Lett. 94, 240404 (2005).

[41] D. Gobert, C. Kollath, U. Schollwöck, and G. Schütz, Real-time dynamics in spin-1/2 chains with adaptive timedependent density matrix renormalization group, Phys. Rev. E 71, 036102 (2005).

[42] T. Platini and D. Karevski, Relaxation in the $X X$ quantum chain, J. Phys. A 40, 1711 (2007).

[43] T. Antal, P. L. Krapivsky, and A. Rákos, Logarithmic current fluctuations in nonequilibrium quantum spin chains, Phys. Rev. E 78, 061115 (2008).

[44] J. Lancaster and A. Mitra, Quantum quenches in an $X X Z$ spin chain from a spatially inhomogeneous initial state, Phys. Rev. E 81, 061134 (2010).

[45] L.F. Santos and A. Mitra, Domain wall dynamics in integrable and chaotic spin-1/2 chains, Phys. Rev. E 84, 016206 (2011).

[46] V. Eisler and Z. Rácz, Full Counting Statistics in a Propagating Quantum Front and Random Matrix Spectra, Phys. Rev. Lett. 110, 060602 (2013).

[47] T. Sabetta and G. Misguich, Nonequilibrium steady states in the quantum $X X Z$ spin chain, Phys. Rev. B 88, 245114 (2013).

[48] J. C. Halimeh, A. Wöllert, I. P. McCulloch, U. Schollwöck, and T. Barthel, Domain-wall melting in ultracold-boson systems with hole and spin-flip defects, Phys. Rev. A 89, 063603 (2014).

[49] V. Alba and F. Heidrich-Meisner, Entanglement spreading after a geometric quench in quantum spin chains, Phys. Rev. B 90, 075144 (2014).

[50] See the Supplemental Material at http://link.aps.org/ supplemental/10.1103/PhysRevLett.115.175301, which includes Refs. [51-66], for details on the experimental setup, the analysis of the time-of-flight data, details on the numerical analysis, and a discussion of related theoretical literature.

[51] T. Giamarchi, Quantum Physics in One Dimension, International Series on Monographs in Physics Vol. 121 (Oxford University Press, Oxford, 2004).
[52] G. Vidal, Efficient Simulation of One-Dimensional Quantum Many-Body Systems, Phys. Rev. Lett. 93, 040502 (2004).

[53] A. Daley, C. Kollath, U. Schollwöck, and G. Vidal, Time-dependent density-matrix renormalization-group using adaptive effective Hilbert spaces, J. Stat. Mech. (2004) P04005.

[54] S. R. White and A. E. Feiguin, Real-Time Evolution Using the Density Matrix Renormalization Group, Phys. Rev. Lett. 93, 076401 (2004).

[55] S. Sorg, L. Vidmar, L. Pollet, and F. Heidrich-Meisner, Relaxation and thermalization in the one-dimensional BoseHubbard model: A case study for the interaction quantum quench from the atomic limit, Phys. Rev. A 90, 033606 (2014).

[56] F. Heidrich-Meisner, S. R. Manmana, M. Rigol, A. Muramatsu, A. E. Feiguin, and E. Dagotto, Quantum distillation: Dynamical generation of low-entropy states of strongly correlated fermions in an optical lattice, Phys. Rev. A 80, 041603 (2009).

[57] D. Muth, D. Petrosyan, and M. Fleischhauer, Dynamics and evaporation of defects in Mott-insulating clusters of boson pairs, Phys. Rev. A 85, 013615 (2012).

[58] C. J. Bolech, F. Heidrich-Meisner, S. Langer, I. P. McCulloch, G. Orso, and M. Rigol, Long-Time Behavior of the Momentum Distribution During the Sudden Expansion of a SpinImbalanced Fermi Gas in One Dimension, Phys. Rev. Lett. 109, 110602 (2012).

[59] S. Mandt, A. E. Feiguin, and S. R. Manmana, Relaxation towards negative temperatures in bosonic systems: Generalized Gibbs ensembles and beyond integrability, Phys. Rev. A 88, 043643 (2013).

[60] T. Antal, Z. Rácz, A. Rákos, and G. M. Schütz, Isotropic transverse $X Y$ chain with energy and magnetization currents, Phys. Rev. E 57, 5184 (1998).

[61] M. Panfil, J. De Nardis, and J.-S. Caux, Metastable Criticality and the Super Tonks-Girardeau Gas, Phys. Rev. Lett. 110, 125302 (2013).

[62] T. Fokkema, I. S. Eliëns, and J.-S. Caux, Split Fermi seas in one-dimensional Bose fluids, Phys. Rev. A 89, 033637 (2014).

[63] T. Antal, Z. Rácz, and L. Sasvári, Nonequilibrium Steady State in a Quantum System: One-Dimensional Transverse Ising Model with Energy Current, Phys. Rev. Lett. 78, 167 (1997).

[64] B. Schmittmann and R. K. P. Zia, Statistical Mechanics of Driven Diffusive Systems, Phase Transitions and Critical Phenomena Vol. 17 (Academic Press, London, 1995).

[65] H. Spohn, Long range correlations for stochastic lattice gases in a non-equilibrium steady state, J. Phys. A 16, 4275 (1983).

[66] S. Katz, J. L. Lebowitz, and H. Spohn, Phase transitions in stationary nonequilibrium states of model lattice systems, Phys. Rev. B 28, 1655 (1983).

[67] S. Will, T. Best, U. Schneider, L. Hackermüller, D.-S. Lühmann, and I. Bloch, Time-resolved observation of coherent multi-body interactions in quantum phase revivals, Nature (London) 465, 197 (2010).

[68] S. Trotzky, L. Pollet, F. Gerbier, U. Schnorrberger, I. Bloch, N. V. Prokof'ev, B. Svistunov, and M. Troyer, Suppression 
of the critical temperature for superfluidity near the Mott transition: Validating a quantum simulator, Nat. Phys. 6, 998 (2010).

[69] F. Gerbier, S. Trotzky, S. Fölling, U. Schnorrberger, J. D. Thompson, A. Widera, I. Bloch, L. Pollet, M. Troyer, B. Capogrosso-Sansone, N. V. Prokof'ev, and B. V. Svistunov, Expansion of a Quantum Gas Released from an Optical Lattice, Phys. Rev. Lett. 101, 155303 (2008).

[70] M. Rigol, Finite-temperature properties of hard-core bosons confined on one-dimensional optical lattices, Phys. Rev. A 72, 063607 (2005).
[71] I. Hen and M. Rigol, Strongly Interacting Atom Lasers in Three-Dimensional Optical Lattices, Phys. Rev. Lett. 105, 180401 (2010).

[72] M. Jreissaty, J. Carrasquilla, F. A. Wolf, and M. Rigol, Expansion of Bose-Hubbard Mott insulators in optical lattices, Phys. Rev. A 84, 043610 (2011).

[73] G. Carleo, F. Becca, M. Schiró, and M. Fabrizio, Localization and glassy dynamics of many-body quantum systems, Sci. Rep. 2, 243 (2012).

[74] M. P. Zaletel, R. S. K. Mong, C. Karrasch, J. E. Moore, and F. Pollmann, Time-evolving a matrix product state with long-ranged interactions, Phys. Rev. B 91, 165112 (2015). 